Supporting Information for

Beyond the Laviron Method: A New Mathematical Treatment for Analyzing the Faradaic Current in Reversible, Quasi-Reversible, and Irreversible Cyclic Voltammetry of Adsorbed Redox Species

Jacob Waelder ${ }^{1}$ and Stephen Maldonado ${ }^{1,2 *}$

1. Program in Applied Physics

University of Michigan

Ann Arbor, MI 48109-1055

2. Department of Chemistry

University of Michigan

Ann Arbor, MI 48105-1055 


\section{S1. Contents}

This document contains information that supplements the main text entitled "Beyond the Laviron Method: A New Mathematical Treatment for Analyzing the Faradaic Current in Reversible, Quasi-Reversible, and Irreversible Cyclic Voltammetry of Adsorbed Redox Species". Section S2 details the limits of the $\chi_{A}$ functions with respect to large and small $m$. Section S3 describes why the explicit value of the start potential, $E_{\text {start }}$, does not appear in the final result. Section S4 illustrates how the general algorithm still computes rationale voltammograms for $\alpha>$ 1. Section S5 describes pertinent details on how to perform the calculation of $\chi_{A}$ to generate cyclic voltammograms. Section S6 presents the Matlab code that was used to generate the figures in the main text. Section S7 summarizes the references cited within this text. 


\section{S2. Limiting Cases}

All of the $\chi_{A}$ expressions in the main text that are written as functions of incomplete gamma functions have two interesting limiting cases for cathodic (forward) sweeps when $m$ approaches either (1) zero or (2) - - . For expediency, the derivation presented here uses eq 9 from the main text for illustration, although the same steps apply to eqs 20, and 21. Since eq 11 features an upper incomplete gamma function, it will be discussed separately after the limits for eq 9 are presented.

\section{Limits for Expressions with Lower Incomplete Gamma Functions}

$E q 9$ of the main text can be written with the substitution $u=-\frac{q}{k_{B} T}\left(E_{a p p}-E^{0}\right)$.

$$
\chi_{A}=\frac{-m^{m+1} \Gamma_{\text {lower }}\left(-m, m e^{u}\right)}{e^{-m e^{u}} e^{-m u}}
$$

The limits with respect to $m$ are difficult to understand without the following relation. Lower incomplete gamma functions have a useful identity.

$$
\Gamma_{\text {lower }}\left(x_{1}, x_{2}\right)=x_{2}^{x_{1}} e^{-x_{2}} x_{1}^{-1} M\left(1,1+x_{1}, x_{2}\right)
$$

In this identity, $M\left(1,1+x_{1,} x_{2}\right)$ is the Kummer function, a confluent hypergeometric function of the first kind with three arguments. ${ }^{1}$ In general, any Kummer function $M(a, b, z)$ is defined in the following way,

$$
M(a, b, z)=\sum_{n=0}^{\infty} \frac{(a)_{n} z^{n}}{(b)_{n} n !}
$$

where $(a)_{n}$ and $(b)_{n}$ are rising factorials. For the Kummer function, the explicit form of $M\left(1,1+x_{1}, x_{2}\right)$ is given below. ${ }^{2}$

$$
M\left(1,1+x_{1}, x_{2}\right)=1+\frac{x_{2}}{\left(1+x_{1}\right)}+\frac{x_{2}{ }^{2}}{\left(1+x_{1}\right)\left(2+x_{1}\right) 2}+\frac{x_{2}{ }^{3}}{\left(1+x_{1}\right)\left(2+x_{1}\right)\left(3+x_{1}\right) 6} \ldots
$$

Combining this form of the Kummer function with the identity for the lower incomplete gamma function and eq 9 yields the following expression. 


$$
\chi_{A}=\frac{-m^{m+1}\left(m e^{u}\right)^{-m} e^{-m e^{u}}(-m)^{-1}}{e^{-m e^{u}} e^{-m u}}\left(1+\frac{m e^{u}}{(1-m)}+\frac{\left(m e^{u}\right)^{2}}{(1-m)(2-m) 2}+\frac{\left(m e^{u}\right)^{3}}{(1-m)(2-m)(3-m) 6} \ldots\right)
$$

This expression condenses to just equating $\chi_{A}$ to the Kummer function.

$$
\chi_{A}=1+\frac{m e^{u}}{(1-m)}+\frac{\left(m e^{u}\right)^{2}}{(1-m)(2-m) 2}+\frac{\left(m e^{u}\right)^{3}}{(1-m)(2-m)(3-m) 6} \ldots
$$

It is from this point that the two limits can be understood.

Limit \#1. $m \rightarrow 0$

When $m$ goes to zero, the higher order terms of the Kummer function are increasingly negligible. Therefore the following statement is true.

$$
\chi_{A} \approx 1+m e^{u}
$$

Since $m$ is small, this expression is a Taylor approximation $\left(e^{x} \approx 1+x\right)$ of an exponential function in the following way.

$$
\chi_{A} \approx e^{m e^{u}}
$$

The result $\chi_{A}=e^{m e^{u}}$ is precisely the expression one would obtain for the irreversible case, where $k_{b}=0$. That is, when $k_{b}$ is zero, eqs 2 and 3 of the main text simplify to the following.

$$
\frac{d \chi_{A}}{d E_{a p p}}=\frac{-1}{v} k_{f} X_{A}
$$

The solution to this differential equation, when $k_{f}$ is evaluated with the Butler-Volmer formalism, is given below.

$$
X_{A}=e^{\frac{-1}{v} \int_{E_{s a a r}}^{E_{a p p}} k_{f} d E_{a p p}}=e^{\frac{k^{0} k_{B} T}{q v \alpha} e^{\frac{-q \alpha}{k_{B} T}\left(E_{a p p}-E^{0}\right)}}=e^{m e^{u}}
$$

Limit \#2. $m \rightarrow-\infty$

When the magnitude of $m$ becomes large, the middle term of the Kummer function also is large. As a result, the Kummer function takes a limiting form. ${ }^{3}$ 


$$
M\left(1,1+x_{1}, x_{2}\right)=\frac{1+x_{1}}{1+x_{1}-x_{2}}
$$

Inserting this form of the Kummer function back into the expression for $\chi_{A}$ results in the following.

$$
\chi_{A}=1+\frac{m e^{u}}{(1-m)}+\frac{\left(m e^{u}\right)^{2}}{(1-m)(2-m) 2}+\frac{\left(m e^{u}\right)^{3}}{(1-m)(2-m)(3-m) 6} \ldots \approx \frac{1-m}{1-m-m e^{u}}
$$

This expression can be simplified in the following manner.

$$
\chi_{A} \approx \frac{1-m}{1-m-m e^{u}}=\frac{1}{1-\frac{m e^{u}}{1-m}}
$$

Since the magnitude of $m$ is much larger than 1, this expression can be further simplified.

$$
\chi_{A} \approx \frac{1}{1-\frac{m e^{u}}{1-m}} \approx \frac{1}{1-\frac{m e^{u}}{-m}}=\frac{1}{1+e^{u}}
$$

Now $\chi_{A}$ is no longer dependent on any rate parameter. This expression is identical to the Fermi function and what is predicted if the surface concentrations are bound by the Nernst relation, i.e. at thermodynamic equilibrium.

$$
\frac{\chi_{A}}{\chi_{A^{-}}}=e^{\frac{q}{k_{B} T}\left(E_{a p p}-E^{0}\right)}
$$

Since $\chi_{A}+\chi_{A^{-}}=1$, the following rearrangement is possible.

$$
\begin{aligned}
& \frac{\chi_{A}}{1-\chi_{A}}=e^{\frac{q}{k_{B} T}\left(E_{a p p}-E^{0}\right)} \\
& \chi_{A}=\frac{e^{\frac{q}{k_{B} T}\left(E_{a p p}-E^{0}\right)}}{1+e^{\frac{q}{k_{B} T}\left(E_{a p p}-E^{0}\right)}}=\frac{1}{e^{-\frac{q}{k_{B} T}\left(E_{a p p}-E^{0}\right)}+1}=\frac{1}{e^{u}+1}
\end{aligned}
$$

\section{Limits for Expressions with Upper Incomplete Gamma Functions}

The steps for finding the limits of eq 11 are more numerous than for eq 9 but the spirit of the derivation is the same. 
$E q 11$ is written below and features an upper incomplete gamma function, where the substitution $u$ ' helps compact the essential form of the expression.

$$
\chi_{A}=\frac{-m^{\prime-m^{\prime}} \Gamma_{\text {upper }}\left(1+m^{\prime},-m^{\prime} e^{\frac{q}{k_{B} T}\left(E_{a p p}-E^{0}\right)}\right)}{e^{m^{\prime} \frac{q}{k_{B} T}\left(E_{a p p}-E^{0}\right)} e^{m^{\prime} e^{\frac{q}{k_{B} T}\left(E_{a p p}-E^{0}\right)}}}=\frac{-m^{\prime-m^{\prime}} \Gamma_{u p p e r}\left(1+m^{\prime},-m^{\prime} e^{u^{\prime}}\right)}{e^{m^{\prime} u^{\prime}} e^{m^{\prime} e^{u^{\prime}}}}
$$

Upper incomplete gamma functions have a related but different identity than lower incomplete gamma functions. Specifically, upper incomplete gamma functions are special cases of Tricomi functions, a confluent hypergeometric function of the second kind $\left(U\left(1,1+x_{1}, x_{2}\right)\right){ }^{4}$

$$
\Gamma_{\text {upper }}\left(x_{1}, x_{2}\right)=x_{2}^{x_{1}} e^{-x_{2}} U\left(1,1+x_{1}, x_{2}\right)
$$

With this identity, the expression for $\chi_{A}$ simplifies somewhat.

$$
\begin{gathered}
\chi_{A}=\frac{-m^{\prime-m^{\prime}}}{e^{m^{\prime} u^{\prime}} e^{m^{\prime} e^{u^{\prime}}}}\left(-m^{\prime} e^{u^{\prime}}\right)^{1+m^{\prime}} e^{m^{\prime} e^{u^{\prime}}} U\left(1,2+m^{\prime},-m^{\prime} e^{u^{\prime}}\right) \\
\chi_{A}=-m^{\prime} e^{u^{\prime}} U\left(1,2+m^{\prime},-m^{\prime} e^{u^{\prime}}\right)
\end{gathered}
$$

Now the two limiting cases for eq 11 can be understood.

Limit \#1. $m \rightarrow 0$

When $m$ approaches 0 , both the second and third arguments in the Tricomi function can attain limiting values. The resultant form of $\chi_{A}$ depends on which limiting value is reached first. For example, when $m$ is sufficiently small so that the second argument assumes a value of 2 , the following identity holds. ${ }^{5}$

$$
\begin{gathered}
U\left(1,2, x_{2}\right)=x_{2}^{-1} \\
\chi_{A}\left(E_{a p p}\right) \approx-m^{\prime} e^{u^{\prime}} U\left(1,2,-m^{\prime} e^{u^{\prime}}\right)=-m^{\prime} e^{u^{\prime}} \frac{1}{-m e^{u^{\prime}}}=1
\end{gathered}
$$

In this instance, the experimental is so much faster than the rate of reduction of $A$ to $A^{-}$that no reaction occurs and $\chi_{A}$ remains unchanged.

If $e^{u^{\prime}}$ is so large (i.e. at large applied overpotentials) that the third argument of the Tricomi function approaches infinity faster than $m$ approaches 0 ,

$$
U\left(1,2+x_{1}, \infty\right)=0
$$




$$
\chi_{A}\left(E_{a p p}\right) \approx-m^{\prime} e^{u^{\prime}} U\left(1,2+x_{1}, \infty\right)=0
$$

In this case, this statement confirms that there is always a large enough overpotential value to drive the reaction to completion, irrespective of $m$.

Limit \#2. $m \rightarrow-\infty$

Tricomi functions are not defined by an asymptotic series but can be approximated to the one shown below. ${ }^{6}$ Unlike the Kummer function, this asymptotic series is not universally valid.

$$
U\left(1, x_{1}, x_{2}\right) \approx \frac{1}{x_{2}} \sum_{n=0}^{\infty} \frac{\left(2-x_{1}\right)_{n}}{\left(-x_{2}\right)^{n}}=\frac{1}{x_{2}}\left[1+\frac{\left(2-x_{1}\right)}{\left(-x_{2}\right)}+\frac{\left(2-x_{1}\right)\left(3-x_{1}\right)}{\left(-x_{2}\right)^{2}}+\frac{\left(2-x_{1}\right)\left(3-x_{1}\right)\left(4-x_{1}\right)}{\left(-x_{2}\right)^{3}} \ldots\right]
$$

Using this approximation, the expression for $\chi_{A}$ again becomes an asymptotic series.

$$
\chi_{A}\left(E_{a p p}\right)=-m^{\prime} e^{u^{\prime}} U\left(1,2+m^{\prime},-m^{\prime} e^{u^{\prime}}\right) \approx 1+\frac{\left(-m^{\prime}\right)}{\left(m^{\prime} e^{u^{\prime}}\right)}+\frac{\left(m^{\prime}\right)\left(1-m^{\prime}\right)}{\left(m^{\prime} e^{u^{\prime}}\right)^{2}}+\frac{\left(m^{\prime}\right)\left(1-m^{\prime}\right)\left(2-m^{\prime}\right)}{\left(m^{\prime} e^{u^{\prime}}\right)^{3}} \ldots
$$

When the magnitude of $m$ is large, the series takes the following form.

$$
\chi_{A}\left(E_{\text {app }}\right) \approx 1-\frac{1}{e^{u^{\prime}}}+\frac{1}{\left(e^{u^{\prime}}\right)^{2}}-\frac{1}{\left(e^{u^{\prime}}\right)^{3}} \ldots
$$

This series has the same form as the expansion of the function $(1+x)^{-1}$.

$$
\frac{1}{1+x}=1-x+(x)^{2}-(x)^{3}
$$

Accordingly, $\chi_{A}$ is no longer dependent on any rate parameter.

$$
\chi_{A} \approx \frac{1}{1+e^{-u^{\prime}}}
$$

This expression for $\chi_{A}$ is exactly the same as described for the limiting case of $m \rightarrow$-o for eq 9 described above, noting that the definitions of $u$ and $u$ ' are related in the following way.

$$
u=-u
$$

\section{S3. Why $\boldsymbol{u}_{\text {start }}\left(E_{\text {start }}\right)$ Does Not Appear in the Final Result}


The solution to the integral of the denominator from eq 7 of the main text will eliminate all terms that explicitly refer to the start potential of the experiment.. The general form of the denominator can be compacted down once the Butler-Volmer formalism is introduced by using the $m$ and $u$ substitutions outlined in the main text.

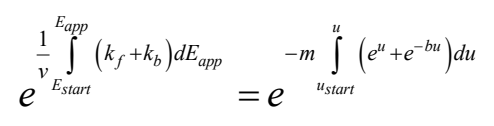

Performing the integration results in the following form for the denominator.

$$
e^{-m \int_{\text {satur }}^{u}\left(e^{u}+e^{-b u}\right) d u}=e^{-m\left(\left(e^{u}-e^{u_{\text {start }}}\right)-\frac{1}{b}\left(e^{-b u}-e^{-b b_{\text {start }}}\right)\right)}
$$

The denominator can then be written as the product of four separate exponential terms.

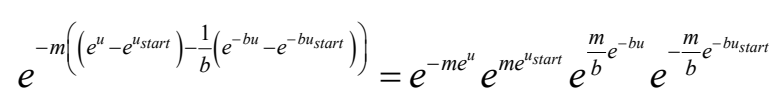

Two of these exponentials are just constants, i.e. they are not functions of $u$ (or $E_{a p p}$ ). For clarity, they are re-positioned as the right most exponentials below.

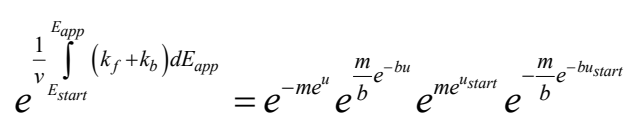

Since the denominator appears in the numerator of $e q 7$ in the main text, the following statement is true.

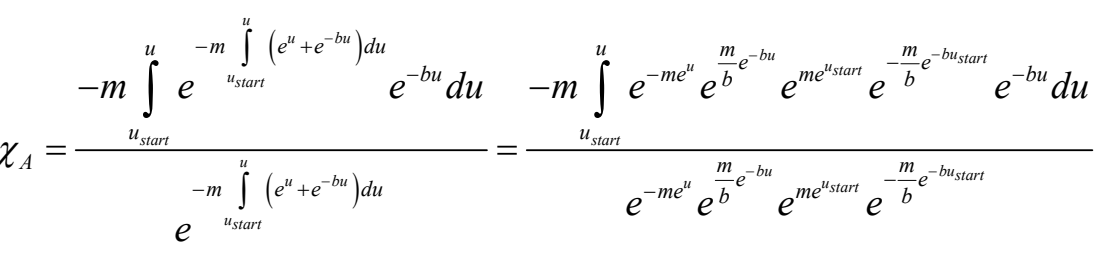

The constant terms can be pulled out of the integral in the numerator and then cancel those terms in the denominator to yield the following result, which is identical to eq 13 in the main text. The function inside of the integral does not depend on the initial start value $\left(u_{\text {start }}=-\frac{q}{k_{B} T}\left(E_{\text {start }}-E^{0}\right)\right)$. 


$$
\chi_{A}=\frac{-m e^{m e^{u_{\text {start }}}} e^{-\frac{m}{b} e^{-b b_{\text {start }}}} \int_{u_{\text {start }}}^{u} e^{-m e^{u}} e^{\frac{m}{b} e^{-b u}} e^{-b u} d u}{e^{-m e^{u}} e^{\frac{m}{b}} e^{-b b} e^{m u_{\text {sart }}} e^{-\frac{m}{b} e^{-b u_{\text {start }}}}}=\frac{-m \int_{u_{\text {start }}}^{u} e^{-m e^{u}} e^{\frac{m}{b} e^{-b u}} e^{-b u} d u}{e^{-m e^{u}} e^{\frac{m}{b} e^{-b u}}}
$$

Choosing $u_{\text {start }}=-\infty$ for the lower bounds of the integral in the numerator is convenient in terms of the final result for $\chi_{A}\left(E_{a p p}\right)$ being written with a single incomplete gamma function and in terms of being consistent with the initial condition, i.e. $\chi_{A}\left(E_{a p p}\right)=1$. 


\section{S4. Behavior of the General Algorithm for $\alpha>1$}

Although $\alpha$ is necessarily bound between 0 and 1 for elementary redox reactions, it is possible to encounter experimental scenarios where $\alpha$ appears larger than 1 when other chemical and/or electrochemical reactions are coupled with the rate-determining elementary redox reaction. Although analysis of such voltammetric data requires some knowledge of the operative mechanism, the general algorithm is in principle amenable for such development. The plots below show the forms of the output of the algorithm based off eqs 20 and 21 of the main text with different $\alpha$ values greater than 1 . No effort was made to connect such plots to a physical description of a particular system. The point here was to demonstrate the algorithm still works in this mathematical space.

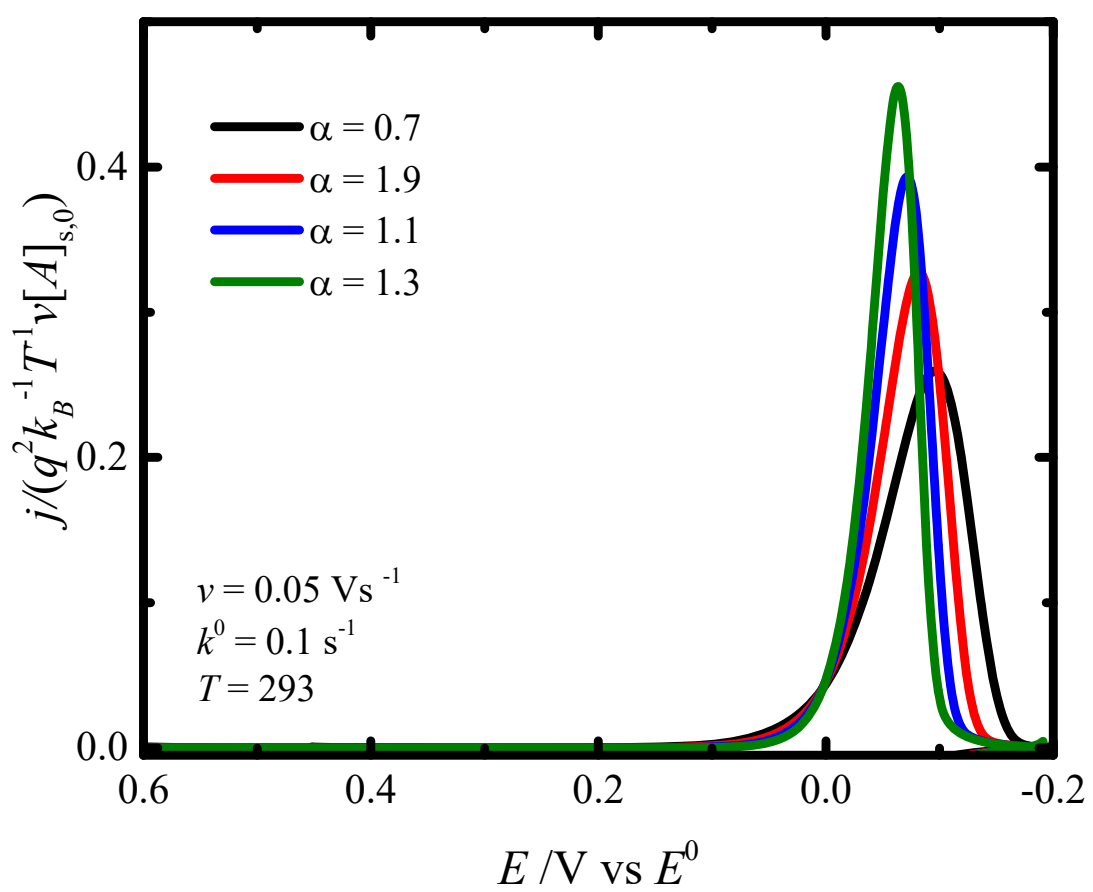

Figure S1. Test of the general algorithm for $\alpha$ values greater than 1. Other simulation parameters: $k^{0}=0.1 \mathrm{~s}^{-1}, T=$ $293 \mathrm{~K}, n=1$, and $v=0.05 \mathrm{~V} \mathrm{~s}^{-1}$

In these plots, the general effect of increasing $\alpha$ is to shift the peak closer to $E^{0}$ and narrow the width. The result is that the peak height exceeds the peak heights for $\alpha=1$ shown in the main text. Finer potential step sizes were necessary to avoid the calculation artifacts described in Section S5 but otherwise no other difficulties were encountered during calculation. 


\section{S5. Details and Comments on Performing the Calculations for $\chi_{A}$}

In this work, all calculations were performed on a personal computer with an Intel i7$8750 \mathrm{H} \mathrm{CPU}$ running at $2.20 \mathrm{GHz}$ with $32 \mathrm{~GB}$ of RAM on Windows $10 \mathrm{Home}$ version 20H2. Base MATLAB version R2021a with the Symbolic Math Toolbox was used throughout. A copy of the custom Matlab code, with annotations, is presented in the next section.

In this section, three common errors that highlight the requirements and limitations of the method we used for the calculations are highlighted.

\section{Coarse Step Size Error}

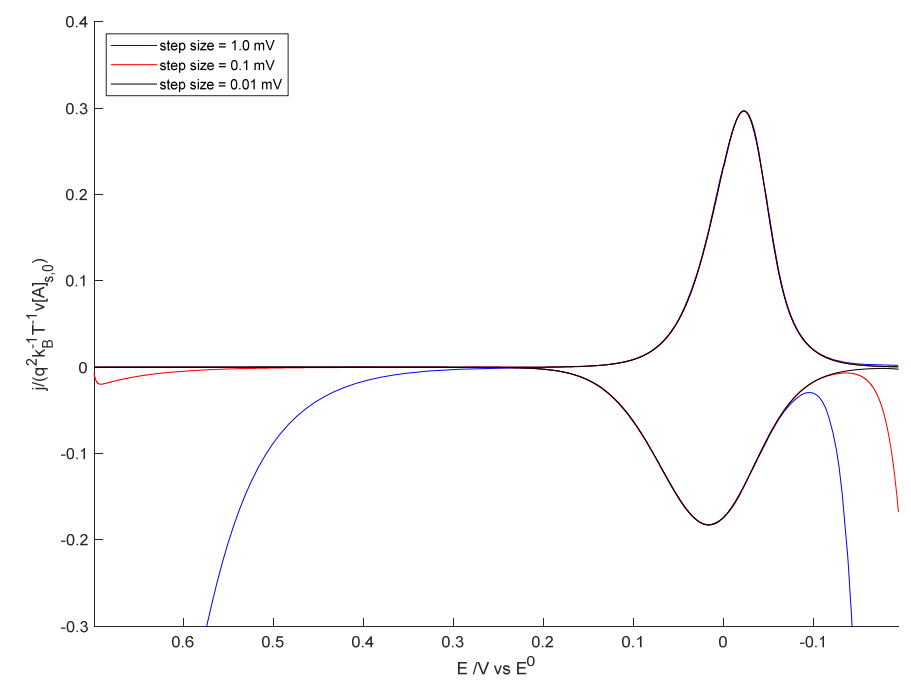

Figure S2. Comparison of the effect of integration step size on the accuracy of the calculated normalized voltammogram. Simulation parameters: $\alpha=0.9, k^{0}=1 \mathrm{~s}^{-1}, T=293 \mathrm{~K}, n=1$, and $v=0.04 \mathrm{~V} \mathrm{~s}^{-1}$ (i.e. $m=0.7015$ )

Figure S2 presents an overlay of full cyclic voltammograms calculated using eqs 20 and 21 of the main text but with different integration step sizes. In the work presented here, all numerical integration was performed with the trapezoidal integration method. Accordingly, the integration step size affected the accuracy of the evaluation of the numerical integral. The accuracy of trapezoidal method is necessarily improved by decreasing the step size. Insufficiently spaced steps resulted in the calculated current density incorrectly increasing at $E_{a p p}$ values much greater or much less than $E^{0}$. Figure $\mathrm{S} 2$ illustrates this point for potential step sizes of 1, 0.1, and $0.01 \mathrm{mV}$. Only the latter step size eliminated the numerical error. A disadvantage with the $0.01 \mathrm{mV}$ step size was an increase in the total calculation time. Notably, the numerical integration error only affects 
the accuracy far from $E^{0}$. The accuracy (and precision) of the calculated current density values near $E^{0}$ were unaffected to the step size, allowing coarser step size for initial calculations to gauge voltammetric shape.

\section{Large $m$ Error}

Calculation of a voltammogram near the reversible limit, i.e. with large $m$, can introduce a separate type of error. Specifically, the incomplete gamma functions used to calculate $\chi_{A}$ generate large numbers when their arguments are large. The igamma algorithm used in Matlab to calculate incomplete gamma functions loses accuracy and the output oscillates between large positive and negative numbers. This error cannot be ameliorated with a small potential step size. An example of this type of artifact is shown in Figure S3. This normalized voltammogram was plotted with a small step size of $0.01 \mathrm{mV}$. However, this numerical error typically occurs outside of the immediate potential range where the voltammetric shape occurs. The $\mathrm{x}$-axis offset that the oscillatory artifact occurs relative to the peak of the reversible voltammogram depends on $m$, with larger $m$ values decreasing the lateral offset from the voltammogram.

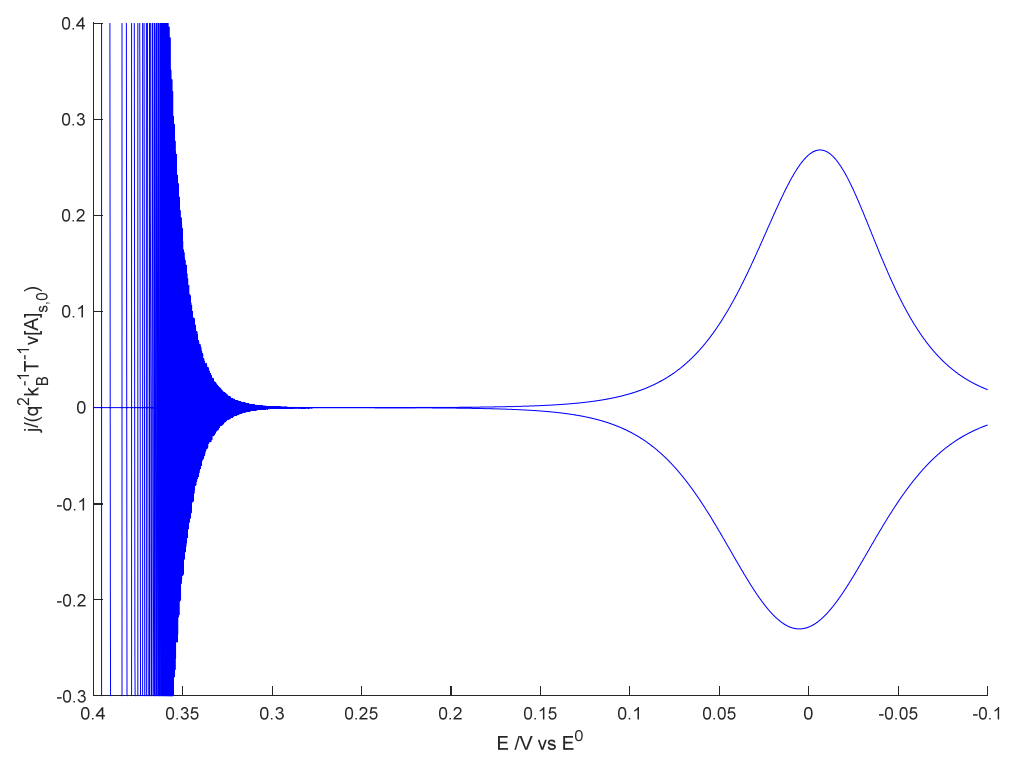

Figure S3. Illustration of the error encountered when $m$ is sufficiently large to yield reversible voltammograms. Simulation parameters: $\alpha=0.9, k^{0}=0.1 \mathrm{~s}^{-1}, T=293 \mathrm{~K}, n=1$, and $v=0.001 \mathrm{~V} \mathrm{~s}^{-1}$ (i.e. $m=2.8059$ ) 


\section{Calculation Limit}

A final calculation limitation occurs with the use of the igamma function in Matlab. Figure S4 highlights the issue. At large overpotentials $(\sim 0.25 \mathrm{~V})$ past the peak of the voltammogram, the second argument of the incomplete gamma functions becomes large and the calculations do not return a real number. In practice, this error has no consequence on obtaining the full shape of the voltammogram, since the current will effectively converge to zero before the calculation limitation is reached.

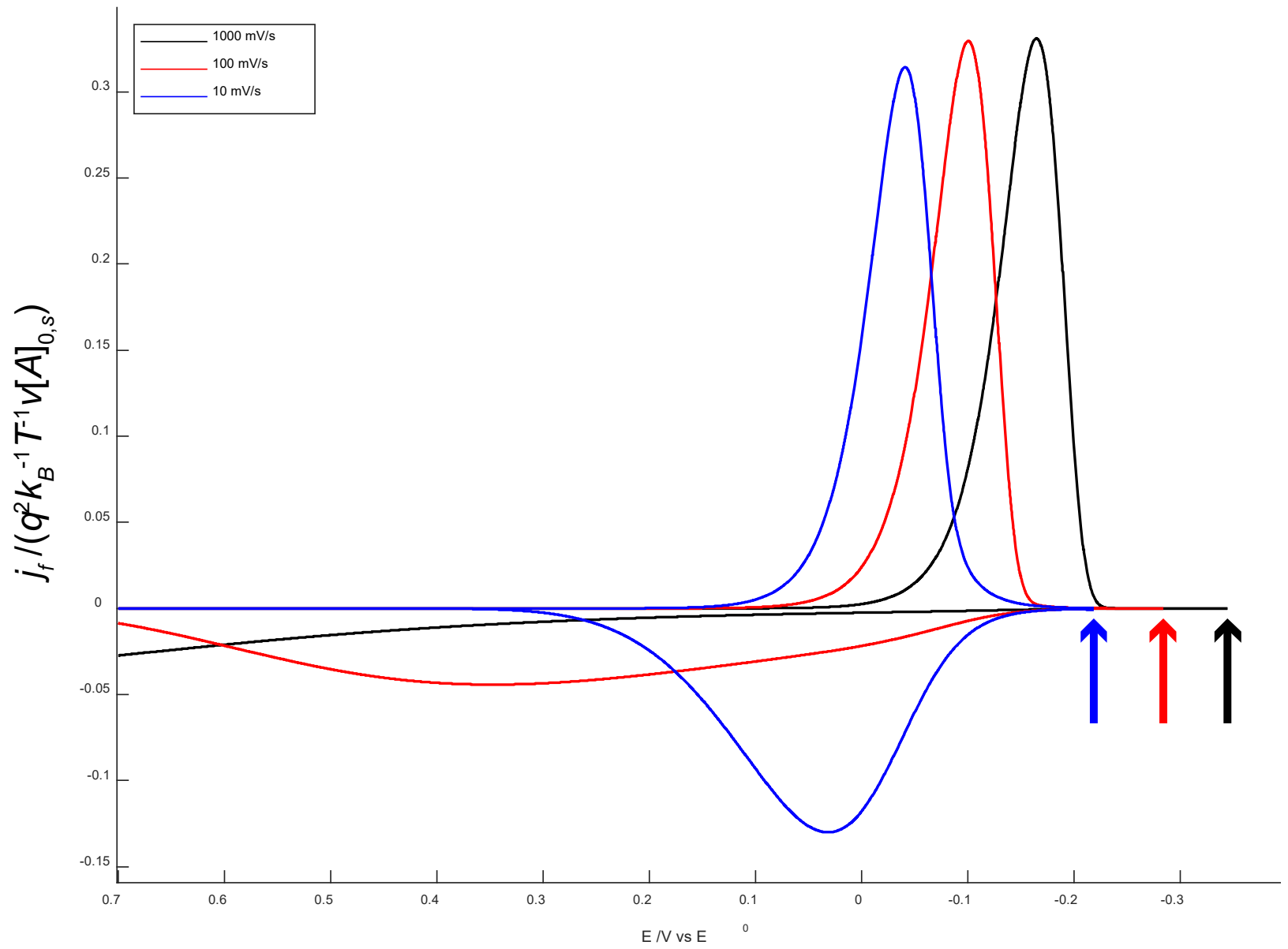

Figure S4. The colored arrows denote the last current value that could be calculated on the forward sweep of each respective calculation. Simulation parameters: $\alpha=0.9, k^{0}=0.1 \mathrm{~s}^{-1}$, and $T=293 \mathrm{~K}$. 


\section{S6. Matlab Code}

Matlab was chosen over Excel for these calculations because in Excel the incomplete gamma functions must be calculated indirectly through the following relations:

$$
\begin{aligned}
& \Gamma_{\text {lower }}\left(x_{1}, x_{2}\right)=\Omega\left(x_{2}, x_{1}, 1, \text { true }\right) / \Gamma\left(x_{1}\right) \\
& \Gamma_{\text {upper }}\left(x_{1}, x_{2}\right)=\Gamma\left(x_{1}\right)-\Gamma_{\text {lower }}\left(x_{1}, x_{2}\right)
\end{aligned}
$$

$\Omega\left(x_{2}, x_{1}, 1\right.$, true $)$ and $\Gamma\left(x_{1}\right)$ are the gamma distribution (GAMMA.DIST) and complete gamma (GAMMA) functions in Excel, which are pre-packaged in every Microsoft Office installation since Office 2013. Together, these functions can give the lower incomplete gamma function. However, a disadvantage with this approach is that these functions require that both arguments are positive. The arguments of the lower incomplete gamma functions can be negative for certain values of $b$ and $m$. To set up an Excel spreadsheet in the most straightforward manner possible, an alternative algorithm should be used for calculation of the incomplete gamma functions (e.g. References 15, 16 , or 27 in the main text). This can be done through a $3^{\text {rd }}$ party add-in or by defining a new custom function directly in Excel.

For readers familiar with Matlab, the explicit code used to generate the figures in the main text is copied below underneath the dashed line.

$\% \%$ REQUIRED PARAMETERS \%\%

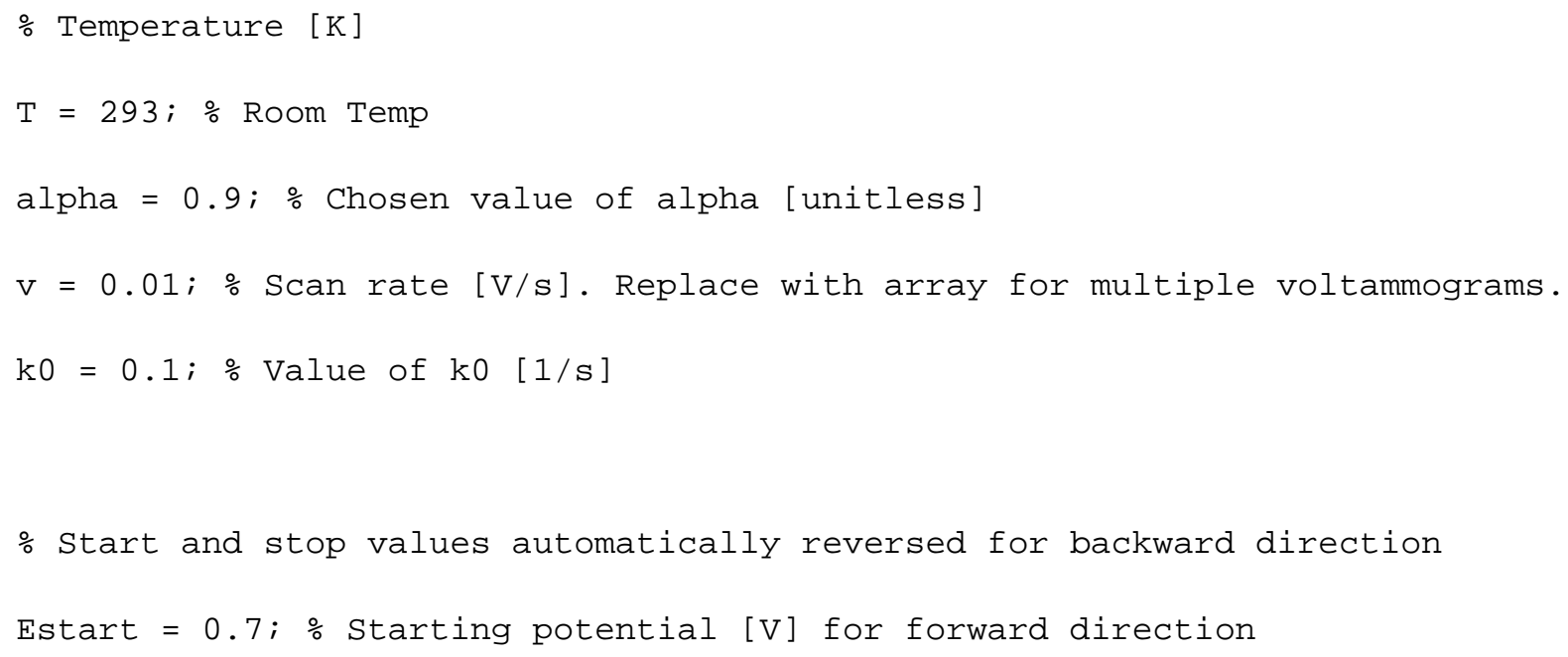


Estop $=-\odot .7 ; \%$ Stopping potential [V] for forward direction

\% Potential step size increments. Smaller values will significantly $\%$ increase computation time, but improve accuracy. Step size of $1 \mathrm{e}-5$ \% typically gives good results $\sim 20$ seconds per voltammogram. Estep $=0.001 ; \%$ Step Size $[\mathrm{V}]$

$\%$ limits y-axis of plot. Helpful when endpoints blow up to infinity ylimit $=\left[\begin{array}{lll}-0.3 & 0.4\end{array}\right]$

\% DO NOT EDIT BELOW THIS LINE

$\%$ Constants

k_B $=1.381 \mathrm{e}-23 ; \%$ Boltzmann constant $[\mathrm{J} / \mathrm{K}]$

$q=1.6023 e-19 ; \%$ Charge of electron $[\mathrm{C}]$

$q k t=q . /\left(k \_B . * T\right) ; \%$ Combine constants for simplicity

$\mathrm{b}=(1$ - alpha) ./ alpha; \% Defined for algebraic simplicity

\% Generate arrays for E_app - E^®. Forward and reverse must be separate.

E_for = linspace $($ Estart, Estop, abs(Estart - Estop)./Estep + 1);

E_rev = linspace (Estop, Estart, abs(Estop - Estart)./Estep + 1);

\% Initialize empty arrays for Chi function and current density.

ChiA_for_final $=\operatorname{zeros}\left(\right.$ length $(v), \operatorname{length}\left(E_{-}\right.$for $\left.)\right)$;

ChiA_rev_final $=\operatorname{zeros}\left(\right.$ length $(v), \operatorname{length}\left(E_{-}\right.$for $\left.)\right)$;

j_for_final $=\operatorname{zeros}\left(\operatorname{length}(v), \operatorname{length}\left(E_{-}\right.\right.$for $\left.)\right)$; 
j_rev_final $=\operatorname{zeros}\left(\operatorname{length}(v), \operatorname{length}\left(E_{-}\right.\right.$for $\left.)\right)$;

\% Main Calculation

for $k \mathrm{k}=1$ :length(v) \% Optional loop for multiple scan rate voltammograms

\%\% Forward Sweep Calculation

tic; \% Start timing clock

k_f $=k \odot . * \exp (-q k t$ * alpha * E_for $) ; \%$ Forward rate constant

$\mathrm{k} \_b=k \odot . * \exp (q \mathrm{kt} . *(1-\mathrm{alpha}) . *$ E_for $) ; \%$ Backward rate constant

$v(k k)=-v(k k) ; \%$ Flips sign of "v" for forward sweep (pos to neg)

$\mathrm{m}=\mathrm{k} \odot . /\left(\mathrm{qkt} .{ }^{*}\right.$ alpha .* $\left.\mathrm{v}(\mathrm{kk})\right)$; \% Define "m" for given parameters

$\mathrm{u}=$-qkt . * alpha . * E_for; \% Simplify $\mathrm{x}$-axis for readability

$u \odot=u(1) ; \%$ Value of "u" when E_app - E^® = E_start

gamma_for $=\operatorname{gamma}(-(b+m))-$ igamma $\left(-(b+m), m .{ }^{*} \exp (u)\right) ; \%$ low inc. gamma

gamma_for $\odot=\operatorname{gamma}(-(b+m))-\operatorname{igamma}\left(-(b+m), m .{ }^{*} \exp (u \odot)\right) ; \%$ eval @zero

$\operatorname{bigG}=\exp \left((m \cdot / b) \cdot{ }^{*}\left(\exp \left(-b \cdot{ }^{*} u\right)+\left(b \cdot{ }^{*} u\right)\right)\right) ; \% G(u)$ in text

$\operatorname{bigG\odot }=\exp \left((\mathrm{m} . / \mathrm{b}) \cdot{ }^{*}\left(\exp \left(-\mathrm{b} \cdot{ }^{*} \mathrm{u} \odot\right)+\left(\mathrm{b} \cdot{ }^{*} \mathrm{u} \odot\right)\right)\right) ; \% \mathrm{G}(\odot)$

littleg $=m \cdot{ }^{*}\left(1-\exp \left(-b \cdot{ }^{*} u\right)\right) \cdot{ }^{*} \exp \left((m \cdot / b) \cdot{ }^{*}\left(\exp \left(-b \cdot{ }^{*} u\right)+\left(b \cdot{ }^{*} u\right)\right)\right) ; \% g(u)$

integral $=\operatorname{cumtrapz}\left(u,(\right.$ gamma_for $\left.) .{ }^{*} \operatorname{littleg}\right) ; \%$ Numerical integral

\% Construct Chi_A in smaller terms for readability

coeff $=-m \cdot \wedge(m+b+1) ; \%$ Coefficient for Chi_A 


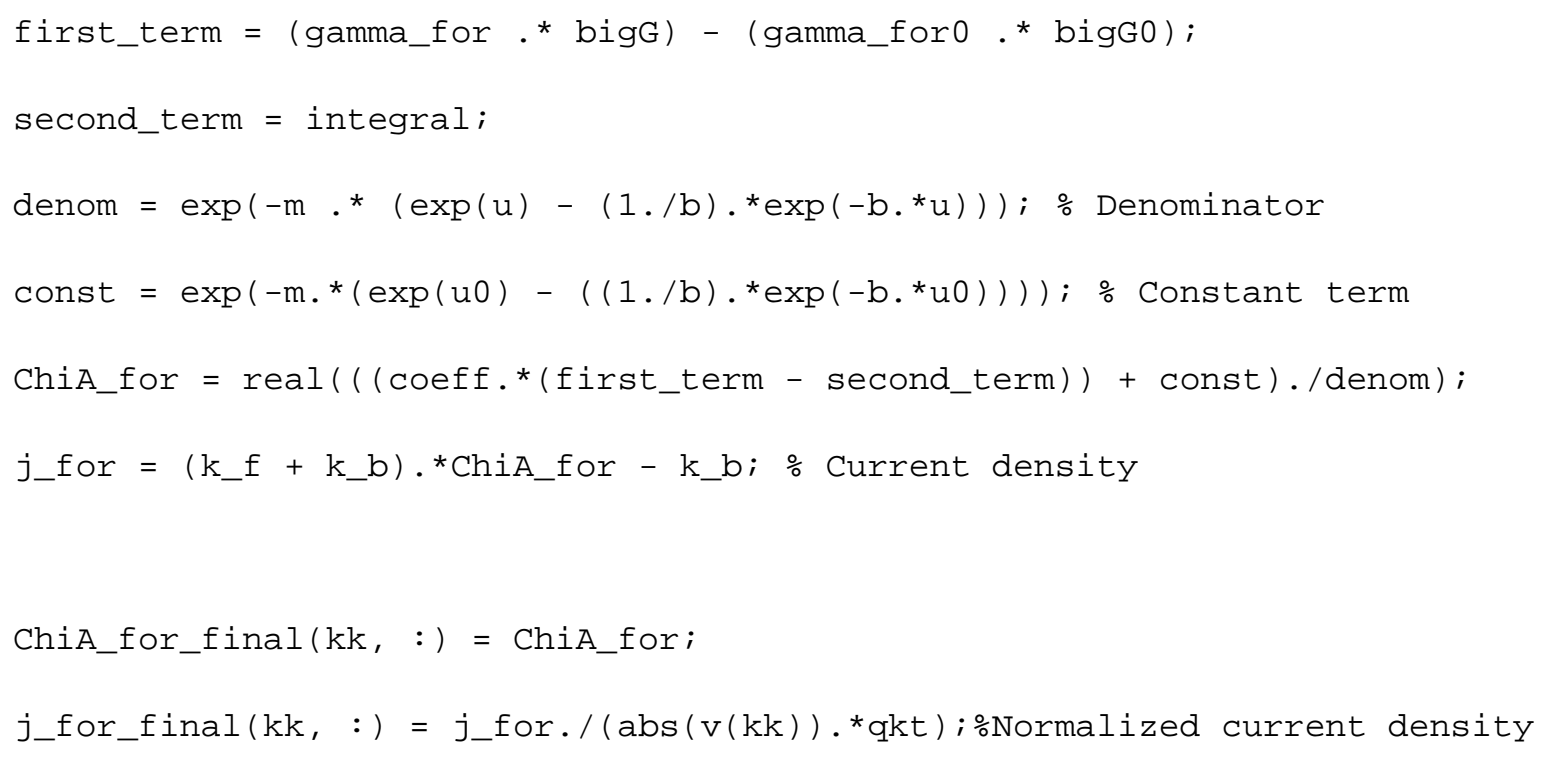

\% Neccessary to calculate with reversed arrays, hense recalculation of \% above terms with "E_rev" integral $=$ cumtrapz $(u$, gamma_rev .* littleg $) ; \%$ Numerical integral 


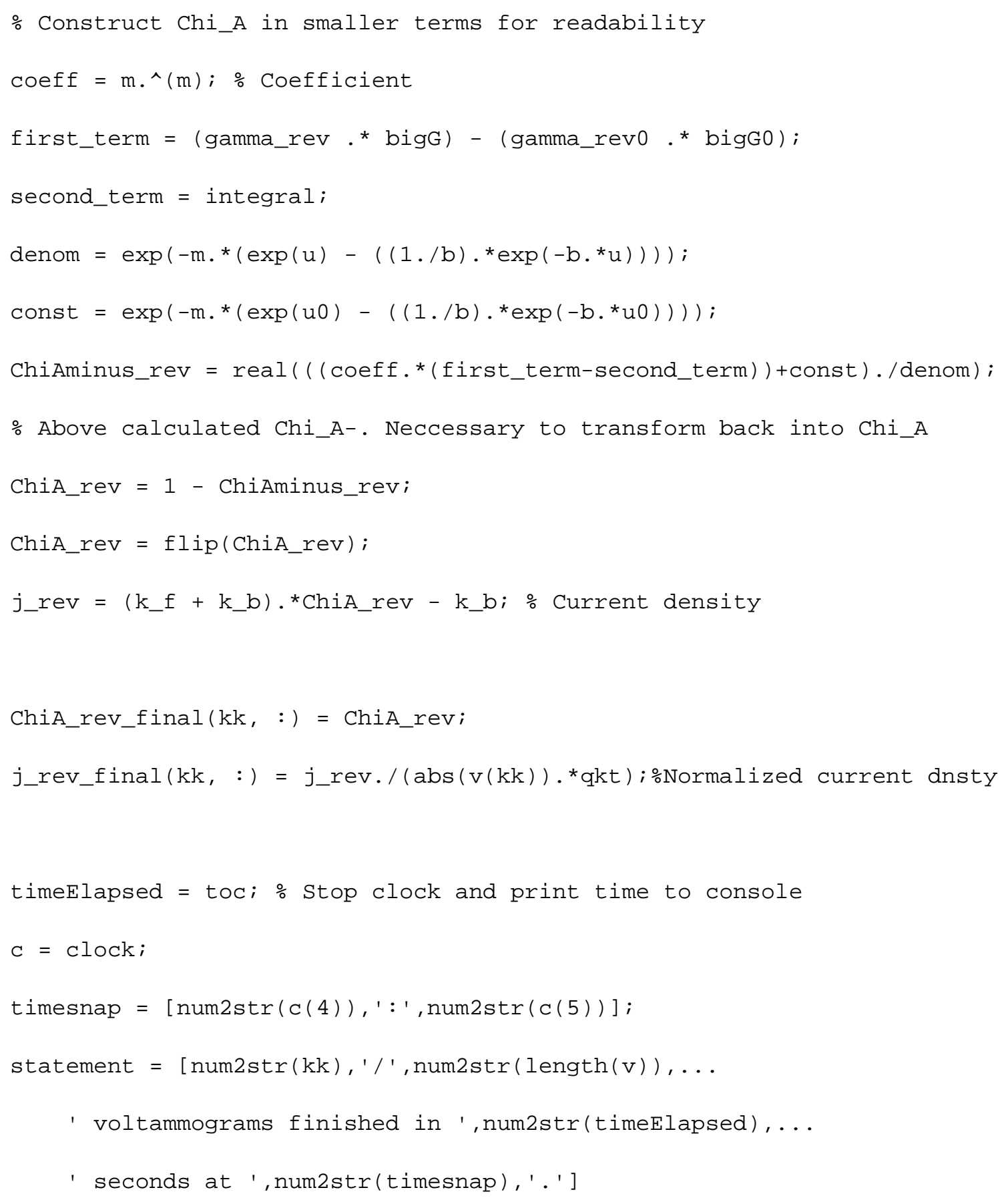

end

\% Plot both forward and backward current densities 
figure;

hold on;

p1 = plot (E_for, j_for_final(1, : ), 'color', 'k');

p2 = plot (E_for, j_rev_final(1, : ), 'color', 'k');

set(gca, 'XDir', 'reverse');

title(['Model, \alpha $={ }^{\prime}, \operatorname{num} 2 \operatorname{str}($ alpha $),{ }^{\prime} \mathrm{k}^{\wedge}\{\Theta\}={ }^{\prime}, \operatorname{num} 2 \mathrm{str}(\mathrm{k} \Theta), \mathrm{s}^{\wedge}\{-1\}, \mathrm{m}=$ ', num2str $(\mathrm{m})]$,$) ;$

xlabel( 'E_1 $\left.-E^{\wedge}\{\Theta\}^{\prime}\right)$;

ylabel( $\left.j\left(E_{-}{ }^{1}\right) '\right)$;

$y \lim (y \operatorname{limit})$; 


\section{S7. References.}

1. Oldham, K. B.; Myland, J. C.; Spanier, J. An Atlas of Functions; 2 ed.; Springer-Verlag New York, 2009, Chapter 47

2. Oldham, K. B.; Myland, J. C.; Spanier, J. An Atlas of Functions; 2 ed.; Springer-Verlag New York, 2009, p. 486

3. Oldham, K. B.; Myland, J. C.; Spanier, J. An Atlas of Functions; 2 ed.; Springer-Verlag New York, 2009, p. 492

4. Oldham, K. B.; Myland, J. C.; Spanier, J. An Atlas of Functions; 2 ed.; Springer-Verlag New York, 2009, Chapter 48

5. Oldham, K. B.; Myland, J. C.; Spanier, J. An Atlas of Functions; 2 ed.; Springer-Verlag New York, 2009, p. 500

6. Oldham, K. B.; Myland, J. C.; Spanier, J. An Atlas of Functions; 2 ed.; Springer-Verlag New York, 2009, p. 502 